\title{
Generation of star-shaped human induced astrocytes with a mature inflammatory phenotype for CNS disease modeling
}

\author{
Dimitrios Voulgaris ${ }^{1,2}$, Polyxeni Nikolakolpoulou ${ }^{2}$ and Anna Herland ${ }^{1,2^{*}}$ \\ ${ }^{1}$ Division of Micro and Nanosystems, KTH Royal Institute of Technology, Stockholm, Sweden \\ ${ }^{2}$ AIMES, Center for Integrated Medical and Engineering Science, Department of Neuroscience, Karolinska \\ Institute, Solna, Sweden \\ *Address correspondence to anna.herland@ki.se \\ Summary
}

Generating astrocytes from induced pluripotent stem cells has been hampered by either prolonged differentiation -spanning over two months -or by shorter protocols that generate immature astrocytes, devoid of salient inflammation-associated astrocytic traits pivotal for CNS neuropathological modeling. We directed human neural stem cells derived from induced pluripotent stem cells to astrocytic commitment and maturity by orchestrating an astrocytic-tuned culturing environment. In under 28 days, the generated cells express canonical and mature astrocytic markers, denoted by the expression of AQP4 and, remarkably, the expression and functionality of glutamate transporter EAAT2. We also show that this protocol generates astrocytes that encompass traits critical in CNS disease modeling, such as glutathione synthesis and secretion, upregulation of ICAM-1 and a cytokine secretion profile which is on par with primary astrocytes. This protocol generates a multifaceted astrocytic model suitable for CNS in vitro disease modeling and personalized medicine through brain-on-chip technologies.

\section{Keywords}

Astrocytes, hiAstrocytes, human induced pluripotent stem cells, hiPSCs, neural stem cells, EAAT2, neuroinflammation, glutathione, ICAM-1.

\section{Introduction}

Astrocytes have long been considered as merely cellular scaffolds - i.e., the brain's glue. Recent studies point out that astrocytes have a prominent role in health and disease; once considered a recycler of neurotransmitters and a supply cabinet for neurons, this star-shaped cell type has an evergrowing role in health and disease (Araque et al., 1999; Barres, 2008; Liddelow et al., 2017).

Astrocytes are a truly versatile brain cell type, regulating a plethora of cellular processes such as glutamate clearance, provision of antioxidants to neurons and relay of inflammatory signals. Additionally, astrocytes actively participate and regulate synaptic transmission (Araque et al., 1999; Perea, Navarrete and Araque, 2009), and they are involved in the pathophysiology of numerous degenerative diseases (Liddelow et al., 2017). 
Importantly, mouse and human astrocytes are transcriptionally and functionally different (Zhang et al., 2016), human astrocytes are larger and have more elaborate processes than mouse astrocytes (Oberheim et al., 2009). Another notable difference is the expression of glutamate transporters EAAT1 and EAAT2; in mouse, GLAST (the mouse equivalent of EAAT1) expression precedes GLT-1 (the mouse equivalent of EAAT2), the latter denotes a postnatal phenotype. Conversely, both transporters are expressed prenatally in humans(DeSilva et al., 2012, p. 2). Additionally, pathological conditions, such as multiple sclerosis (MS), affect humans exclusively('t Hart, 2016) and experimentally induced mouse models fail to deepen our knowledge in potential treatments (Sriram and Steiner, 2005). Stem cell technology paved the way for the generation of numerous differentiation protocols that generate human induced astrocytes (hiAstrocytes), bypassing the need for primary sources, and bringing forth human models that can potentially capture in higher biofidelity human astrocytes than mouse models.

Astrocytes respond to pathological conditions in a disease-specific fashion; in ALS, the glutamate transporter EAAT2 is lost in astrocytes suggesting a causal relationship between neuronal excitotoxicity and EAAT2 loss in astrocytes(Rothstein et al., 1995). Apart from inflammatory cytokine secretion upon stimulation (e.g., IL-6 and IL-8 (Choi et al., 2014)), astrocytes can also express ICAM-1 in pathological conditions (Frohman et al., 1989) such as MS(Brosnan et al., 1995), brain injury and AD (Müller, 2019). In MS, ICAM-1 on astrocytes serves as direct communication with infiltrating leukocytes (Héry et al., 1995; Lee et al., 2000, p. 1) and microglia(Akiyama et al., 1993). Astrocytes are also unique shapeshifters; they remodel their processes and orient them towards lesions (Schiweck, Eickholt and Murk, 2018) while in transgenic AD animals, astrocytes exhibit reduced complexity in their processes (Rodríguez et al., 2009). In epilepsy, astrocytic processes appear thicker and longer (Oberheim et al., 2008). Astrocytes synthesize and secrete glutathione (GSH)(Yudkoff et al., 1990; Sagara, Makino and Bannai, 1996) shielding CNS cells from oxidative stress, which is also reflected in the high astrocytic GSH content $\sim 8 \mathrm{mM}$ (Dringen and Hamprecht, 1998). Interestingly, GSH availability is altered in TBI (Harris et al., 2012) and autism(Rose et al., 2012; Gu et al., 2013), while GSH efflux is impaired in AD(Lovell, Xie and Markesbery, 1998; Sultana and Butterfield, 2004). It remains to be determined whether GSH disturbance is the etiology, contributing factor or simply an effect of pathological conditions. Arguably, astrocytes have indeed a prominent role in pathological conditions of the CNS.

The need to model interactions of the CNS necessitates reliable and sustainable (i.e., non-primary) sources of human astrocytes. The extent of information extracted from an in vitro study is limited by the model used; hence, a holistic view of astrocytes in pathology demands the concurrent existence of a spectrum of traits that exceed the current traits of astrocytic differentiations. To recapitulate pathological conditions in vitro, an astrocytic model, among others, should: 1) express and have functionally active EAAT1 and EAAT2 transporters 2) capacity for an immune response upon inflammatory stimuli both via cytokine secretion and upregulation of inflammatory adhesion markers (i.e., ICAM-1) that is comparable to primary astrocytes and 3) synthesize and secrete GSH that is comparable to primary astrocytes.

There is a lack of a short astrocytic differentiation protocol ( $<30$ days) from a stable precursor that can recapitulate all the mentioned processes. Currently, astrocyte generation is hampered by either extensive differentiations (>60 days) (Roybon et al., 2013; Holmqvist et al., 2015; Oksanen et al., 2017, p. 1; Perriot et al., 2018) or by shortened differentiation protocols ( $<60$ days) that lack a process-bearing phenotype and GSH synthesis/secretion (Santos et al., 2017). Our recent efforts in astrocyte generation yielded GFAP-negative cells that lack EAAT2 functionality, thus resembling an immature phenotype 
(Lundin et al., 2018, 2020). We envisioned that we could generate mature astrocytes with in vivo-like morphology and functionality by providing an astrocytic-tuned milieu to neural stem cells. An astrocytogenic milieu comprises a suitable differentiation media, an astrocytic-tuned ECM coating and appropriate cell-to-cell contact (Li et al., 2019).

Almost all astrocyte differentiations in a monolayer format have been carried out in coatings such as Matrigel or laminins. However, none of these ECM molecules are used for in vitro culturing of human primary astrocytes. An interesting candidate ECM molecule is collagen; collagen is indeed not abundant in the bulk ECM of the CNS (Zimmermann and Dours-Zimmermann, 2008) and is mainly restricted in the basement membrane and meninges. In vitro studies unveiled that astrocytes express fibrillar collagen, which is inhibited in vivo by EGF signaling and meningeal cells (Heck et al., 2003, 2007). Interestingly, when fetal astrocytes were cultured in a 3D collagen gel, they assumed a star-like morphology instead of the elongated phenotype that is ubiquitous in a conventional culturing environment (Placone et al., 2015). As opposed to laminins, collagen is not well suited for neuronal differentiations (Ma et al., 2008), hence creating an ECM environment that can potentially boost astrocytic commitment and dampen the neurogenic potential of neural stem cells. Gelatin, a denatured form of collagen, has been used for the primary isolation of astrocytes(Sinyuk and Williams, 2020); thus we elected to use it in our differentiation strategy.

Here, we report on a differentiation strategy that unleashes the astrocytic potential of iPS-derived neural stem cells without any shorting steps. By day 28, human iPS-derived astrocytes (hiAstrocytes) feature a star-shaped morphology, display inflammatory potency and functional uptake of both astrocytic glutamate transporters (EAAT1 and EAAT2) as well as an mRNA and protein profile that resembles human astrocytes. Additionally, we demonstrate that hiAstrocytes synthesize and secrete glutathione. This astrocytic model can be used for CNS disease modeling since it encompasses a multitude of traits that are altered during pathological conditions.

\section{Results}

\section{Neural stem cells generate star-shaped cells in 28 days under astrocytogenic conditions}

Neuroepithelial cells (NES) are an intermediate cellular stage derived from induced pluripotent stem cells that can generate both neurons and astrocytes. NES can be cryopreserved and cultured up to 100 passages without major phenotype changes and are therefore a robust starting point for neural differentiation(Falk et al., 2012). We used three iPS-derived neural stem cell lines, NES C9, NES C7 and NES AF22. Two of these lines (NES C9 and NES AF22) lines have been previously used for astrocyte differentiation (Lundin et al., 2018, 2020). Here, we differentiated the neural stem cells for 28 days (Figure 1a) using a primary astrocytic (AM) media that has been previously shown to induce astrocytic traits in various research groups (Tcw et al., 2017; Soubannier et al., 2020). In this approach, the growth factor cocktail is tailored for astrocytic growth and maintenance (Michler-Stuke, Wolff and Bottenstein, 1984; Haselbacher et al., 1989; Codeluppi et al., 2011). 
Neural stem cells were transiently cultured in suspension as aggregates and further differentiated in a monolayer format. Cells were seeded at $288 \mathrm{~K} /$ well in a 6 -well plate format (day -1 ) in $0.2 \%(\mathrm{w} / \mathrm{v}$ ) gelatincoated wells in N2B27 media. The day after (Figure 1b, day 0, NES C9), most cells were in suspension, forming small aggregates. Media was carefully removed, and cells were washed once with DPBS ( $w / \mathrm{Ca}^{++}$ and $\mathrm{Mg}^{++}$) before adding AM media supplemented with AGS and FBS.

The day after (Figure 1b, day 0), the cell aggregates had attached to the culture vessels, and we passaged (at $30 \mathrm{~K} / \mathrm{cm} 2$ ) the cultures after 1-2 days upon reaching confluency since that is pivotal for the morphology of hiAstrocytes. The first 6 days are crucial for a successful astrocyte differentiation; cells assume a colony-like morphology, growing outwards. By day 7, cells lost their NES-like behavior; namely, cells did not grow in NES colonies upon passaging, appeared more elongated, and a small percentage of cells assumed a triangular morphology (Figure 1b, day 7). By day 14, the frequency of triangular-shaped cells increased (Figure 1b, day 14) while the growth rate decreased (compared to NES), suggesting a switch to differentiation over self-replication. By day 21, cellular processes could be detected emanating from the somata, resembling star-shaped morphologies. The proliferation rate was further reduced by day 26 (Figure $1 \mathrm{~b}$, day 26), and cells transitioned to more noticeable morphological changes assuming a star-like morphology (Figure 1b, day 27), apparent in all lines (Figure 1c, i) NES C9 ii) NES C7 and iii) AF22).

\section{HiAstrocytes have a distinct astrocytic mRNA expression profile that differs from spontaneously differentiated cells.}

We characterized hiAstrocytes by RT-qPCR and compared them to human fetal cortical astrocytes (HFA) at low passage (p.3). In hiAstrocytes C9, the so-called gliogenic switch, Nuclear factor IA (NFIA)(Tchieu et al., 2019), was upregulated during the differentiation while the neuronal progenitor marker $D C X$ was downregulated (Figure 2a), denoting glial commitment. Most astrocytic markers such as CD44, GFAP and ALDH1L1 were on par with the expression levels in HFA, while AQP4 and S100B were significantly enriched in hiAstrocytes opposed to HFA (Figure 2b). Interestingly, NES and HFA had the same expression level of the astrocytic marker S100B. HiAstrocytes C7 and AF22 had an expression pattern that was comparable to hiAstrocytes C9 (Figure 2c).

Next, we sought to unveil the transcriptomic differences between the hiAstrocytes, and cells generated by spontaneous differentiation of the NES lines. Spontaneously differentiated cells (SDCs) were generated by growth factor withdrawal for 28 days (Figure S1a). NFIA was, strikingly, upregulated in all cell lines in both hiAstrocytes and SDCs, while CD44 expression was restricted to the hiAstrocytes (all lines) and SDCs AF22 (Figure 2c). As expected, DCX was upregulated in all lines in SDCs; conversely, $D C X$ was downregulated in hiAstrocytes (all lines). Glial Acidic Fibrillar Protein (GFAP) was highly upregulated in hiAstrocytes (all lines), while GFAP expression varied in SDCs. SDCs in AF22 showed higher upregulation of GFAP than SDCs C9 or C7. The differential expression of GFAP and CD44 in SDCs suggests a mixture of neurons and glia in SDCS AF22 while there is a neuronal enrichment in SDCS C9 and C7.PCA of 19 genes analyzed by RT-qPCR showed a distinct separation between SDCs and hiAstrocytes, denoting the impact of the differentiation strategy on enriching astrocytic fate (figure 2d). hiAstrocytes clustered closer to HFA than NES or SDCs. Specifically, hiAstrocytes C7 and AF22 clustered closer to HFA than 
hiAstrocytes C9. NES and SDCs had distinct clusters further away from HFA. Hierarchical clustering revealed that indeed hiAstrocytes from all three lines clustered with HFA and were distinct from SDCs.

We also assessed the regionality of NES and hiAstrocytes. Gene expression analysis of two regionality markers RELN and SLIT1, revealed that, strikingly, the NES lines have a different dorsoventral identity (Figure 2e). While all lines had no or insignificant expression of RELN, NES C7 seems to have a distinct regional identity denoted by the SLIT1 expression, which remained almost unchanged during astrocyte differentiation, denoting a regionally patterned precursor that gives rise to specifically VA3 astrocytes. In hiAstrocytes C9, RELN and SLT1 were upregulated, while in hiAstrocytes AF22, there was a very low expression ( $R E L N \mathrm{Ct}=33$, SLIT: $\mathrm{Ct}=32)$. HFA also had a similar RELN expression pattern $(\mathrm{Ct}=32)$, while SLIT1 was not detected.

\section{Quantification of astrocytic markers and processes shows a superior proteomic and phenotypic profile compared to HFA.}

We performed immunocytochemistry and stained hiAstrocytes C9, C7, AF22 and HFA for the common astrocytic markers S100B, CD44, GFAP, AQP4 ALDH1L1 and the cytoskeletal marker VIMENTIN. All hiAstrocytes lines and HFA stained positive for CD44 (Figure 3a) while ICC quantification all models reached almost $100 \% \mathrm{CD}_{4} 4^{+}$cells (Figure 3b). HiAstrocytes $\mathrm{C9}, \mathrm{C7}$, AF22 and HFA did not stain for DCX

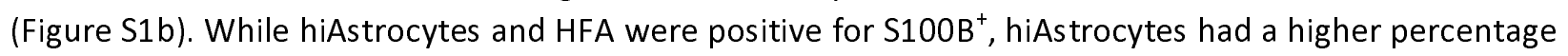

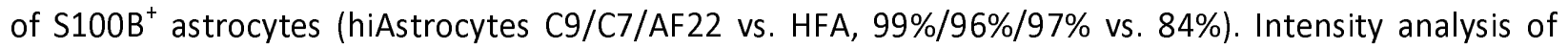
each population showed that hiAstrocytes $\mathrm{C} 9$ and $\mathrm{C} 7$ populations had higher average intensity than HFA, as denoted by the shift in the violin plots (Figure S1c).

HFA were $24 \%$ GFAP $^{+}$while hiAstrocytes C9, C7 and AF22 were $20 \%, 46 \%$ and $14 \%$ GFAP $^{+}$, respectively. Quantification of ALDH1L1 revealed a low percentage (16\%) of positive cells while the quantification among the three lines was not coherent, with hiAstrocytes $\mathrm{C} 7$ having the higher percentage of positive cells (83\%) followed by hiAstrocytes C9 (64\%) and AF22 (49\%). HiAstrocytes from all three lines were positive for AQP4 with almost $100 \%$ positive cells (hiAstrocytes C9/C7/AF22, 96\%/98\%/96\%), while HFA had a lower percentage of AQP4-positive cells (16\%).

For the quantification of astrocytic processes, we stained cells with VIMENTIN. HiAstrocytes C9 showed the highest percentage (37\%) of multiple processes ( $>=5$, Figure $3 c$ ), followed by hiAstrocytes $C 7(20 \%)$ and AF22 (13\%) (>=5, Figure 3d). Only $2 \%$ of HFA exhibited multiple processes ( $>=5$, Figure $3 d$ ).

\section{HiAstrocytes exhibit functional EAAT1- and EAAT2-mediated glutamate uptake}

We next sought to characterize the glutamate uptake capacity of hiAstrocytes, a critical in vivo functionality of astrocytes. NES showed an uptake of $7.25 \mathrm{nmol}$ per million cells while hiAstrocytes showed more than a 10 -fold increase in glutamate uptake than NES $(80.57 \mathrm{nmol}$ per million cells, $\mathrm{p}<0.0001$ ). HiAstrocytes showed four times higher glutamate uptake rate compared to HFA (20.03 nmol per million cells, $p<0.0001$ )

We used the non-substrate compounds UCPH1 and WAY213613 to inhibit the two astrocytic glutamate transporters EAAT1 and EAAT2, respectively. Both inhibitors significantly blocked glutamate uptake in hiAstrocytes compared to vehicle (vehicle vs. UCPH1, 80.57 vs. $7.13 \mathrm{nmol}$ per million cells, $p<0.0001$ and 
vehicle vs. WAY213613, 80.57 vs. $52.95 \mathrm{nmol}$ per million cells, $\mathrm{p}=0.0057)$. Even though the GLAST inhibition (UCPH1) lowered glutamate uptake compared to vehicle (vehicle vs. UCPH1, 20.03 vs 5.16 nmol per million cells), it was not significant $(p=0.3118)$. HFA did not exhibit functional EAAT2 with the WAY213613 inhibitor.

Further characterization of those transporters through RT-qPCR and ICC revealed that in hiAstrocytes SLC1A2, the gene encoding for the EAAT2 transporter was highly upregulated (Figure 4b) compared to HFA, which showed a slight downregulation (hiAstrocytes vs. HFA, $\Delta \Delta C \mathrm{Ct} 6.46$ vs. -1.12 ). The transporter SLC1A3 had almost the same expression pattern in both cell populations. ICC of the two glutamate transporters showed that both hiAstrocytes and HFA are EAAT1 ${ }^{+}$. HiAstrocytes stained positive for EAAT2 while HFA were weakly stained (Figure 4c).

\section{HiAstrocytes harbor unique inflammatory potency and antioxidant properties.}

A vital aspect of a differentiated cell's repertoire is its capacity to respond to inflammatory agents, which should be on par with the in vivo counterpart, in our case, HFA. That is an important aspect of modeling pathologies in vitro. Hence, we challenged the hiAstrocytes and HFA with IL1- $\beta$ and stained for the intracellular adhesion molecule 1 (ICAM-1) and measured the cytokine secretion of IL- 6 and IL-8, two cytokines that are secreted by astrocytes upon inflammation.

ICC revealed that all lines and HFA showed almost no staining of ICAM-1 when unstimulated, while when incubated with IL1- $\beta$ for 24 hours, most cells were ICAM ${ }^{+}$(Figure $5 a$ ). Specifically, quantification of the ICC showed that in basal conditions, all hiAstrocytes and HFA had less than $10 \%$ ICAM- $1^{+}$(Figure $5 \mathrm{~b}$ ). Upon inflammatory stimuli, hiAstrocytes $\mathrm{C} 9$ and $\mathrm{C} 7$ had the higher percentage of ICAM- ${ }^{+}$cells, $84 \%$ and $83 \%$, respectively. HFA had slightly fewer positive cells, amounting to $75 \%$ ICAM $-1^{+}$. HiAstrocytes AF22 had the lowest number of ICAM-1 ${ }^{+}, 61 \%$.

Cytokine secretion of IL-8 revealed that hiAstrocytes (all lines) and HFA responded to IL-1 $\beta$ by secreting IL8, which was significant compared to basal conditions ( $p>0.0001$, figure $5 c$ ). All lines showed a differentiation-acquired immune potency of IL-8 secretion. HFA and hiAstrocytes C7 had the highest secretion (56 pg and $47 \mathrm{pg}$ per 1000 cells, respectively) of IL-6 (Figure 5d). HiAstrocytes C9 secreted an order of magnitude less, amounting to 8 pg per 1000 cells, while hiAstrocytes AF22 secreted 0.171 pg per 1000 cells. IL-1 $\beta$-challenged HFA, hiAstrocytes C9 and C7 had significantly higher IL-6 secretion compared to their basal conditions ( $p>0.0001$ ), while IL-6 secretion in hiAstrocytes AF22 challenged with IL-1 $\beta$ was not significantly higher compared to their basal conditions ( $p>0.001$, Basal: 0.013 vs. 0.171 pg per 1000 cells).

A largely unexplored astrocytic aspect in stem cell-derived astrocytes is the capacity of astrocytes in vivo to protect the CNS against oxidative stress through glutathione. Astrocytes have a very high glutathione content ( 8mM) (Dringen and Hamprecht, 1998) that they continuously replenish since they constantly provide GSH precursors to neurons and brain endothelial cells(Huang et al., 2020).

We measured the total GSH content of NES, hiAstrocytes and HFA, and we also sampled medium extracellularly to assess 1 ) the intracellular content of hiAstrocytes and how it compared to HFA GSH levels and 2) whether they can export it and thus extend their capacity to protect neighboring cells against reactive oxygen species. 
Our analysis showed that all hiAstrocytes showed significantly higher intracellular total GSH than the undifferentiated NES ( $p<0.0001$, Figure $5 e$ ). When compared to HFA, hiAstrocytes had higher total GSH intracellularly, while HFA had $4.42 \mathrm{nmol}$ total GSH per million cells, hiAstrocytes C9, C7 and AF22 showed $10.33(p<0.0001), 9.18(p<0.0001)$ and $7.68 \mathrm{nmol}(p=0.0014)$ total $\mathrm{GSH}$ per million cells, respectively.

Measurements of total extracellular GSH revealed that all hiAstrocytes exported considerably more total GSH than their NES counterparts. While all NES lines hovered between 1.1-2.2 nmol total GSH per million cells, hiAstrocytes C9, C7 and AF22 showed a remarkable increase ranging from 11.2 to 16.6 ( $p<0.0001$, figure $5 f$ ). Expanding more on that, the total extracellular GSH in hiAstrocytes C9 and AF22 was almost eight times higher than their respective NES lines. HiAstrocytes $C 7$ showed an increase amounting to 10 times more compared to NES C7. Extracellular values of hiAstrocytes were on par with HFA.

\section{Discussion}

Over the past years, there has been a plethora of astrocytic differentiation protocols, some more elaborate and time-consuming than others, spanning over many months or sorting steps(Holmqvist et al., 2015; Santos et al., 2017; Leventoux et al., 2020) . Our differentiation strategy relies on creating an astrocytogenic milieu that brings forth astrocytic traits in only 28 days. The differentiation protocol we report is advantageous for CNS disease modeling, considering the simplicity and relatively cost-effective reagents. We created the astrocytogenic milieu as a combination of ECM component, culture medium and cell-to-cell communication (regulated via seeding density) to generate functional and morphologically star-shaped astrocytes in under 28 days. Collagen does not promote neuronal differentiations and is secreted by astrocytes in vitro. Gelatin, combined with a growth media designed to promote and sustain astrocytes creates an environment that guides NES into functional star-shaped astrocytes.

In our previous work, we compared astrocyte differentiation protocols to adult astrocytes per industry standards(Lundin et al., 2018). However, fetal astrocytes are a more relevant model to compare hiPSderived astrocytes. Adult human astrocytes have undergone a developmental process that is very challenging to recapitulate in vitro; hence, astrocytic differentiations are most likely to generate astrocytes that have developmental proximity to human fetal astrocytes than to adult human astrocytes.

Our differentiation strategy generates astrocytes that resemble HFA both on an mRNA and protein level. HiAstrocytes and HFA are not in the same cluster In the PCA plot, albeit hierarchical clustering reveals that hiAstrocytes cluster with HFA and not with SDCs. We postulate that the reasons behind that deviation are: 1) the different developmental stage, 2) different regionality, and 3) the fact that there is always a caveat with the acute isolation of primary cells (Lange et al., 2012). Additionally, in vitro 
expansion of primary astrocytes follows an adaptation mechanism that may ensue a transcriptomic shift (i.e. dedifferentiation) similar to what has been seen with other CNS primary cells such as brain endothelial cells (Sabbagh et al., 2018).

HiAstrocytes had a higher expression of $S 100 B$ compared to HFA. The S100B enrichment in hiAstrocytes

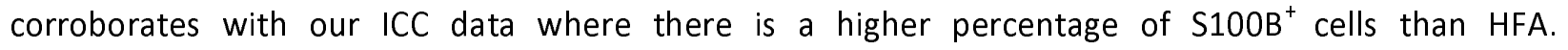
Interestingly, hiAstrocytes had a higher average intensity of $\mathrm{S}_{100 \mathrm{~B}^{+}}$cells (per cell) than HFA. That could denote a developmental stage that surpasses the developmental stage of commercially available HFA (18-20 GW). In mice, $S 10 O B$ expression of $\mathrm{GFAP}^{+}$astrocytes is associated with a mature state that lacks neural stem cell traits (Raponi et al., 2007). In humans, the transition from fetal to mature astrocytes is characterized by S100B upregulation (Zhang et al., 2016), while the higher percentage of AQP4 ${ }^{+}$and $\mathrm{ALDH}_{1 \mathrm{~L} 1^{+}}$in hiAstrocytes reinforces the notion that hiAstrocytes have a developmental stage that transcends the $20 \mathrm{GW}$ developmental stage of HFA (Zhang et al., 2016).

Secondly, a largely unaddressed issue in most hiPS-derived astrocyte protocols is the regionality of astrocytes. Transcriptomic analysis of two regionality markers associated with the spinal cord, RELN and SLIT1 (Hochstim et al., 2008; Rowitch and Kriegstein, 2010; Clarke et al., 2021), revealed that the hiAstrocytes have a distinct regional identity; specifically, hiAstrocytes C9 appeared to be either a mixture of VA1, VA2 astrocytes or VA3. Interestingly, NES C7 was the only NES line that showed expression of SLIT1 which persisted during the differentiation associating hiAstrocytes C7 with ventrally located VA3 population. AF22 HiAstrocytes did not show expression of either RELN or SLIT1, suggesting a more anterior regional identity. HFA did not express any of these markers, as expected since these astrocytes were isolated from the cortex. However, more regionality markers should be explored to pinpoint the regionality of hiAstrocytes accurately. To what extent neural stem cells patterning affects the anteroposterior and dorsoventral identity of hiAstrocytes remains to be seen. Bradly and et al. showed that the regionality of neural stem cells influences the gene expression of downstream differentiation to astrocytes; hence, it follows that astrocytes derived from differentially patterned NES lines cannot possibly cluster very tightly (Bradley et al., 2019) owing to differential expression patterns in various regions of the brain, one notable example is glutamate transporter expression(Bar-Peled et al., 1997) which is region-dependent.

The non-overlapping clusters in the PCA echo the abovementioned differences between hiAstrocytes and HFA; perhaps inclusion of primary astrocytes of different parts of the brain would better associate with hiAstrocytes. Albeit having a different regional identity, all hiAstrocytes stained positive for the canonical astrocytic markers, S100B, AQP4, ALDH1L1 and CD44. Interestingly, the generated cells are GFAP $^{+}$which was not observed in our previous protocol to generate astrocytes from long-term proliferating NES(Lundin et al., 2018, 2020; Lam, Sanosaka, et al., 2019). NES (all lines) did not stain for any of these markers but were positive for VIMENTIN (Figure S2).

The morphology of astrocytes is also another salient feature of astrocytes, mirroring their physiological/pathological state. A star-shaped morphology has been greatly elusive in shorter differentiation protocols (Tcw et al., 2017; Lundin et al., 2018), and only possible to attain after prolonged differentiation $\sim 5$ months as documented by Oksanen and et al.(Oksanen et al., 2017). The hiAstrocytes showed a star-like morphology and had more complex morphologies (processes $>=3,41$ $69 \%$, line depended, Figure S3) than HFA (18\%), making this differentiation strategy ideal for CNS disease modeling where astrocytic processes are affected by pathological conditions(Oberheim et al., 
2008; Rodríguez et al., 2009; Schiweck, Eickholt and Murk, 2018). A noteworthy pathological condition is schizophrenia, where researchers have shown that astrocytes in schizophrenia have a lower number of astrocytic processes than healthy astrocytes (Windrem et al., 2017).

A noticeable difference between the NES lines in SDCs was the different populations they generated. NES C9 and C7 were biased towards neuronal commitment, while NES AF22 generated a mixture of glial and neuronal populations. Our data corroborate with findings from other studies; specifically, NES AF22 has been shown to produce a mixture of glial and neuronal population upon growth factor withdrawal(Lam, Sanosaka, et al., 2019) while NES C7 mainly generated neurons(Lam, Moslem, et al., 2019). Adhering to that paradigm, NES AF22 should have the greatest potential for astrocyte differentiation (compared to NES C7 and NES C9), but strikingly NES AF22 underperformed compared to NES C9 and NES C7. NES AF22 has previously also underperformed compared to other lines when differentiated towards the astrocytic lineage(Lundin et al., 2018).We postulate that these differences could be attributed either to the different neural inductions used to generate the lines (NES C9 and C7: dual-SMAD and NES AF22: spontaneous neural induction) or to the type of reprogramming of the corresponding hiPSC lines (iPS C9 (Uhlin et al., 2017) and C7 (Kele et al., 2016) non-integrating sendai virus and iPS AF22 integrating lentivirus). The former could be a plausible reason since differences in neural inductions has been shown to affect astrocytic potential (Nadadhur et al., 2018); however, more experiments on how neural induction/reprogramming affects downstream differentiations should be done.

EAAT1 expression and functionality has been documented previously in NES-derived astrocytes (Lundin et al., 2018) and constitutes perhaps the bare minimum functional requirement of iPS-derived astrocytes. HiAstrocytes showed specific EAAT1 glutamate uptake and had a similar expression pattern to HFA. EAAT2 is not expressed in HFA in vitro (Lee et al., 2008), and its expression is induced by coculturing primary astrocytes with neurons (Swanson et al., 1997; Schlag et al., 1998) or brain endothelial cells (Lee et al., 2017). Interestingly, our results show that EAAT2 was significantly upregulated in hiAstrocytes than HFA, without using any molecular inducers such as Ceftriaxone (Lee et al., 2008) or coculturing with other CNS cells. The glutamate assay and the specific EAAT2 inhibitor, WAY213613, revealed that EAAT2 transporters in hiAstrocytes are functional. We postulate that the differentiation strategy, more specifically, the high density that cells are kept before each passage and the presence of bFGF in the differentiation media, work synergistically towards EAAT2 expression and functionality. Notch signaling has been shown to increase GLT-1 expression in mice (Lee et al., 2017), and the same has been shown for bFGF (Savchenko et al., 2019). Other groups have shown EAAT2 expression in iPSderived astrocytes (Roybon et al., 2013; Shaltouki et al., 2013; Leventoux et al., 2020), and here we report on an astrocytic model that shows specific EAAT2 expression and functionality.

EAAT2 has gained much attention since studies suggest that excitotoxicity-induced neuronal death is closely correlated with neurological disorders (Pajarillo et al., 2019) such as ALS and AD (Rothstein et al., 1995; Takahashi et al., 2015; Garcia-Esparcia et al., 2018). In autism, the significance of EAAT2 over EAAT1 has been documented in a mouse model (Bristot Silvestrin et al., 2013). EAAT2 expression and functionality is pivotal when attempting to model in vitro these neurological disorders. Ceftriaxone is one candidate that showed promising results in vitro and animal models in increasing GLT-1 expression (Rothstein et al., 2005; Colton et al., 2010). Even though this specific compound failed in clinical trials, compounds that modulate EAAT2 expression are still a plausible therapeutic route for ALS (Rosenblum and Trotti, 2017, p. 2). 
Additionally, hiAstrocytes exhibited strong inflammatory potency. Upon IL-1b simulation, hiAstrocytes secreted IL-6 and IL-8, which is on par with what other groups have shown for hiPS-derived astrocytes (Holmqvist et al., 2015; Santos et al., 2017; Perriot et al., 2018). We quantitatively analyzed and compared cytokine secretion between hiPS-derived astrocytes and primary fetal astrocytes. Our results showed that hiAstrocytes C9 and C7 secreted IL-8 and IL-6 in comparable levels to HFA. Additionally, upon IL-1b stimulation, hiAstrocytes expressed ICAM-1. ICAM-1 upregulation is a link in the relay of inflammatory stimuli between contact-mediated and secreted cytokines, activation of ICAM-1 has been shown to elicit IL-6 secretion (Lee et al., 2000). Additionally, NES (all lines) were not responsive when challenged with IL-1 $\beta$ staining negative for ICAM-1.

The intracellular GSH content of hiAstrocytes and HFA is on par with other astrocytes studies (Figure S3a), ranging from 16 to $50 \mathrm{nmol} / \mathrm{mg}$ protein (Raps et al., 1989; Devesa et al., 1993; Dringen, 2000). HiAstrocytes $C 9$ and $C 7$ hovered around the higher end of that range (57 nmol/mg protein), while hiAstrocytes AF22 and HFA had lower GSH content,35, and $24 \mathrm{nmol} / \mathrm{mg}$ protein, respectively. Our differentiation approach generates astrocytes that exhibit superior export of GSH. One other study that measured extracellular GSH in hiAstrocytes documented $\sim 8.13 \mathrm{nmol} / \mathrm{mg}$ protein (Oksanen et al., 2017) while our study generated astrocytes that exported at least six times more GSH (Figure S3b and c). The rate of GSH export is hiAstrocytes ranged between 2.9 to $3.8 \mathrm{nmol} \mathrm{GSH} /$ ( $\mathrm{h} \times \mathrm{mg}$ protein) (Figure S3d). HFA showed an export rate of $2.9 \mathrm{nmol}$ GSH / ( $\mathrm{h} \times \mathrm{mg}$ protein).

Interestingly, our values are in the range of previous studies in rat primary astrocytes, $3.2 \mathrm{nmol} / \mathrm{h} \mathrm{x} \mathrm{mg}$ protein) (Dringen, Kranich and Hamprecht, 1997). HiAstrocytes C9 and C7 exhibited $\sim 7$ times more GSH export, than their respective NES lines and AF22 10 times more GSH than NES AF22 (in nmol GSH / million cells). This data could suggest that differentiated cells have a proper expression of $A B C C 1$ for GSH export (Renes et al., 1999; Hirrlinger, Schulz and Dringen, 2002; Minich et al., 2006). Astrocytes continuously synthesize GSH; the highest consumption of GSH occurs in the form of GSH export. Hence astrocytes synthesize steady-state GSH to compensate for exported GSH. The intracellular content of GSH is defined by 1) the rate that cells synthesize GSH from precursor molecules and 2) the rate that cells export GSH. Contemplating the similar export rates between hiAstrocytes and HFA and considering that all cells were cultured in the same media (i.e., same availability of GSH precursors), hiAstrocytes showed 2 to 3 times more GSH content in 24 hours, compared to HFA. This difference suggests that HFA lag and cannot fully compensate for the GSH export; hence hiAstrocytes have superior capacity to mitigate insults and reactive oxygen species. The strategic location of astrocytes in the brain, covering over $90 \%$ of the brain vessels with their processes, constitutes astrocytes the first line of defense against xenobiotics and toxins that enter the brain. Neurons are unable to synthesize glutathione on their own and rely on astrocytes as a glutathione source (Wang and Cynader, 2000). Moreover, astrocyte-secreted glutathione counteracts the detrimental effects of an insult to the blood-brain barrier (Huang et al., 2020). ROS are prevalent in many, if not all, neurological conditions (Bains and Shaw, 1997; Cadet and Brannock, 1998; Li et al., 2013; Popa-Wagner et al., 2013; Fang et al., 2017), are they part of the etiology or disease progression? Are astrocytes the cause or the domino factor in disease progression? GSH cycle disturbances can potentially shed light on this front.

We report on specific astrocytic traits that have not been assembled before in a hiPS-derived astrocyte generation. Astrocytes harbor in their immune response a repertoire that transcends mere cytokine secretion; astrocytic responses are characterized by a cascade of reactions that involve morphological rearrangement of their processes and expression of adhesion molecules (e.g., ICAM-1), loss of glutamate 
transporters and glutathione redox balance shift. Consequently, this hiPS-derived astrocytic protocol constitutes a multifaceted in vitro model that may serve as a powerful tool enabling pathological cues to surface and potentially further deepen our knowledge of how astrocytes are involved in the etiology, onset, and progression of CNS pathological conditions. This conveniently short differentiation protocol may contribute to the advancement of personalized medicine and to improved implementations of more complex vitro models such as organ-on-chip technologies.

\section{Acknowledgements}

We thank the iPS core and the Biomedicum Imaging Core (BIC) at Karolinska Institute for access to their service.

We also thank Thomas E. Winkler for sharing his knowledge in statistics and Isabelle Matthiesen for the help with cell culture.

PN and AH acknowledges funding from the Swedish Research Council (2019-01803).

AH acknowledges funding from the Knut and Alice Wallenberg Foundation (no. 2015-0178), Göran Gustafsson Stiftelse, and Forksa utan Djurförsök.

\section{Conflict of Interest}

The authors declare no conflict of interest.

\section{Author contributions}

DV: Conceptualization; methodology; investigation; analysis and visualization; writing - original draft.

PN: Validation; methodology; writing review \& editing

AH: Conceptualization; methodology; resources, writing review \& editing, Supervision and Administration.

\section{Figure legends}

Figure 1 Neural stem cells generate star-shaped cells in 28 days under astrocytogenic conditions

a) Schematic presentation of the duration of the protocols used to derive neuroepithelial stem cells and hiAstrocytes. b) Brightfield images of NES C9 for differentiation days 0,7,14, 21, 26 and 27. Scale bar 100 $\mu \mathrm{m}$. c) Brightfield images of hiAstrocytes C9. (i) hiAstrocytes C7 (ii) and hiAstrocytes AF22 (iii) on day 26 of differentiation. Scale bar $50 \mu \mathrm{m}$. 
Figure 2 HiAstrocytes have a distinct astrocytic mRNA expression profile that differs from spontaneously differentiated cells.

mRNA analysis ( $\triangle C$ Ct values) of a) SOX1, NES, NFIA and $D C X$ and b) astrocyte-specific markers $C D 44$, $S 100 B, A L D H 1 L 1, G F A P$ and AQP4 for hiAstrocytes C9 (purple) and HFA (orange). Data shown from $\mathrm{n}=3$ independent experiments. Error bars represent \pm SD. Statistical analysis was done on the $\Delta C t$ values by using multiple unpaired student's test (Holm-Šídák method) with Welch correction. ${ }^{*} p<0.05$. c) Heatmap mRNA levels ( $\triangle \triangle C t$ ) of hiAstrocytes and SDCs from NES C9, C7 and AF22 d) PCA plot and e) Hierarchical clustering of NES C9, C7 and AF22 along with their corresponding differentiated astrocytes (hiAstro) and spontaneously differentiated cells (SDCS). HFA from one isolation were used. Normalized $\Delta C t$ values were used for the PCA plot and hierarchical clustering.

Figure 3 Quantification of astrocytic markers and processes shows superior proteomic and phenotypic profile compared to HFA.

a) ICC images of astrocytic markers S100B, CD44, GFAP, ALDH1L1 and the cytoskeletal marker VIMENTIN of hiAstrocytes C9, C7 and AF22 along with HFA. Scale bar, $50 \mu \mathrm{m}$. b) quantification of astrocytic markers S100B, CD44, GFAP, AQP4 and ALDH1L1, each dot represents one field of view c) quantification of astrocytic processes, each dot represents the average of four fields of view.

Figure 4 HiAstrocytes exhibit functional EAAT1- and EAAT2-mediated glutamate uptake a) Glutamate uptake assay for NES C9 (orange), hiAstrocytes C9 (purple) and HFA (yellow), $\mathrm{n}=3$ independent wells. Error bars represent \pm SD. Statistical analysis was done using two-way ANOVA followed by Tukey's post-hoc test. $\left.{ }^{*} p<0.05,{ }^{* *} p<0.01, * * * p<0.001, * * * * p<0.0001 \mathrm{~b}\right)$ mRNA analysis of glutamate transporters SLC1A2 and SLC1A3 for hiAstrocytes $C 9$ and HFA, $\mathrm{n}=3$ independent experiments. Error bars represent \pm SD. Statistical analysis was done on the $\Delta C t$ values by using multiple unpaired student's test (Holm-Šídák method) with Welch correction. *p $<0.05$. c) ICC images of glutamate transporters EAAT1 and EAAT2 for HiAstrocytes C9 and HFA.

Figure 5 hiAstrocytes harbor unique inflammatory potency and antioxidant properties a) ICC images of ICAM-1 in hiAstrocytes C9, C7 and AF22 along with HFA under basal and inflammatory conditions (IL-1 $50 \mathrm{ng} / \mathrm{ml}$ for $24 \mathrm{~h}$ ). Scale bar $50 \mu \mathrm{m}$. b) Quantification of ICAM-1 ${ }^{+}$cells under basal (grey) condition or under inflammatory stimuli (IL-1ß $50 \mathrm{ng} / \mathrm{ml}$ for $24 \mathrm{~h}$, blue). Secretion levels of c) IL-8 and d) IL-6 in NES C9, C7 and AF22 and their corresponding astrocyte differentiation (hiAstrocytes C9, C7 and AF22) and HFA under basal (grey) and inflammatory conditions (IL-1 $350 \mathrm{ng} / \mathrm{ml}$ for $24 \mathrm{~h}$, blue), $\mathrm{n}=3$ independent wells. Glutathione levels e) intracellularly and f) extracellularly. NES C9, C7 and AF22 along with their corresponding astrocyte differentiation (hiAstrocytes C9, C7 and AF22) and HFA, $n=3$ independent wells. Error bars represent \pm SD. Statistical analysis was done using two-way ANOVA followed by Tukey's post-hoc test. ${ }^{*} p<0.05, * * p<0.01, * * * p<0.001, * * * * p<0.0001$.

Figure S1 Characterization of SDCs and hiAstrocytes

a) Brightfield images after 28 of spontaneous differentiation of i) NES C9 (SDCs C9) ii) NES C7 (SDCs C7) and iii) NES AF22 (SDCs AF22). Scale bar $100 \mu \mathrm{m}$. b) DCX staining of hiAstrocytes C9, C7, AF22 and HFA. Scale bar $50 \mu \mathrm{m}$. C) Violin plot depicting the average intensity (per cell) of S100B for hiAstrocytes C9, C7, AF22 and HFA. 
Figure S2 ICC of the NES lines used in this study ICC images of NES lines NES C9, C7 and AF22 for the astrocytic markers S100B, CD44, GFAP, ALDH1L1. Scale bar $50 \mu \mathrm{m}$ (20x).

Figure S3 Quantification of astrocytic processes Quantification of astrocytic processes of HiAstrocytes C9 (dark purple), C7 (light purple), AF22 (light purple) and HFA (green). Each dot represents an average of four fields of view (10x).

Figure S4 Characterization of immune potency of NES and quantification of glutathione levels of hiAstrocytes and HFA.

a) ICAM staining for basal and inflammatory conditions for NES C9, C7 and AF22. Scale bar $50 \mu \mathrm{m}$. b) total intracellular and c) total extracellular glutathione content for hiAstrocytes C9, C7, AF22 and HFA normalized by total protein content, $n=3$ independent wells. Error bars represent $\pm S D$. Statistical analysis was done using two-way ANOVA followed by Tukey's post-hoc test. ${ }^{*} p<0.05,{ }^{* *} p<0.01,{ }^{* * *} p<0.001$, $* * * * p<0.0001$. d) comparison with the work from Oksanen et al. (2017), $n=3$ different lines, the dot in the Oksanen study is the mean of the values in the Oksanen study derived by dividing with the molecular weight of glutathione e) Glutathione efflux rate of hiAstrocytes C9, C7, AF22 and HFA.

\section{STAR methods}

All reagents were purchased from Thermo Fisher Scientific, MA, USA, unless otherwise stated.

All processes were done according to the manufacturer's instructions, unless otherwise stated.

\section{Cell culture}

Human Neuroepithelial stem cells (NES)

NES lines (Control 9 and 7: male, dual-SMAD neural induction (Chambers et al., 2009) and AF22: female, spontaneous neural induction (Falk et al., 2012)) were provided by the iPS Core Facility (Karolinska Institute). All lines were cultured and passaged until p.23-24 in DMEM: F12 Glutamax supplemented with N2 1:100, B27 1:1000, $10 \mathrm{ng} / \mathrm{ml}$ bFGF (R\&D Systems, MN, USA) and $10 \mathrm{ng} / \mathrm{ml} \mathrm{EGF} \mathrm{(Sigma} \mathrm{Aldrich,}$ MO, USA) the complete media is termed N2B27 on double coated PLO (20ug/ml Sigma Aldrich, MO, USA) and L2020 (1:500, Sigma Aldrich, MO, USA) flasks. Culture vessels were incubated for 2 hours with PLO washed thoroughly $\mathrm{x} 2$ with DPBS $\left(\mathrm{w} / \mathrm{o} \mathrm{Ca}^{++}\right.$and $\mathrm{Mg}^{++}$) and then coated for at least 2 hours with L2020. After p.23-24, NES were cultured in N2B27 in low EGF $(1 \mathrm{ng} / \mathrm{ml})$.

NES were passaged 1:4-1:5. Briefly, cells were washed with DPBS ( $w / 0 \mathrm{Ca}^{++}$and $\mathrm{Mg}^{++}$) and then incubated with trypLE for 3-4 min. TrypLE was deactivated using equal volumes of DTI and DMEM: F12 glutamax (1:1:1), spun down at 200g and resuspended in N2B27. 
The day after passaging bFGF $(10 \mathrm{ng} / \mathrm{ml}$ ) or EGF (1 or $10 \mathrm{ng} / \mathrm{ml}$, depending on the passage number) was added cultures the day after the media was completely replenished, this motif of cell feeding was done throughout the NES culture.

\section{Astrocyte differentiation}

NES were passaged using trypLE for 3-4 min and trypLE was deactivated by adding DTI. Cells were spun down and resuspended in N2B27 with low EGF $(1 \mathrm{ng} / \mathrm{ml})$. Cells were seeded out in 6-well plates at $288 \mathrm{~K} /$ well. The day after, cells were carefully washed with DPBS $\left(\mathrm{w} / \mathrm{Ca}^{++}\right.$and $\mathrm{Mg}^{++}$) and media was changed to AM medium (with the addition of AGS and FBS, ScienCell, CA, USA). Media changes were done every other day, and cells were passaged 1-2 days upon reaching confluency, except for the first passage where cells were passaged on days 6-7. For all passages, cells were consistently seeded out at $30 \mathrm{~K} / \mathrm{cm}^{2}$.

All astrocyte differentiations were carried out using NES with $p . \#>30$.

Spontaneously differentiated cells (SDCs)

NES (p.\#> 30) were detached using trypLE for 3-4 mins and were seeded out at $30 \mathrm{~K} / \mathrm{cm}^{2}$ in doublecoated PLO (20ug/ml) and L2020 (1:100) in N2B27 (low EGF). The day after, media was changed to DMEM: F12 glutamax with N2 1:100 and B27 1:100. The media was completely changed every other day. Cells were split once during the differentiation (day 7) at $45 \mathrm{~K} / \mathrm{cm}^{2}$ on PLO-L2020 plates. Following the first passage, media was changed every 3 to 4 days. DMEM: F12 glutamax with N2 1:100 and B27 1:100 was first warmed at $37^{\circ} \mathrm{C}$, and then $10 \mathrm{ng} / \mathrm{ml} \mathrm{BDNF}$ and $10 \mathrm{ng} / \mathrm{ml} \mathrm{GDNF}$ (both R\&D Systems, MN, USA) was added to the media prior to complete media change. On day 28 , cells were harvested.

\section{Human Fetal Astrocytes (HFA)}

Human astrocytes were cultured in ScienCell Media AM supplemented with 2\% FBS, 1\% AGS and 1\% P/S. HFA that exceeded passage 7 were not used for experiments. Cells were passaged once they reached confluency with a 1:4 ratio. Briefly, cells were washed with DPBS $\left(\mathrm{w} / \mathrm{o} \mathrm{Ca}^{++}\right.$and $\mathrm{Mg}^{++}$) and then incubated with trypLE for 3-4 min. TrypLE was deactivated using DTI 1:1 (TrypLE:DTI), spun down at 200g and resuspended in complete AM media.

\section{Immunocytochemistry}

Cells were initially washed with DPBS (w/ $\mathrm{Ca}^{++}$and $\mathrm{Mg}^{++}$) and fixed with 4\% PFA (VWR, PA, USA) for 10 min at room temperature. After washing twice with PDBS $\left(\mathrm{w} / \mathrm{Ca}^{++}\right.$and $\left.\mathrm{Mg}^{++}\right)$, cells were incubated with blocking buffer ( $10 \%$ goat serum (Sigma Aldrich, MO, USA) and $0.1 \%$ Triton $\mathrm{X}-100$ in DPBS $\left(\mathrm{w} / \mathrm{Ca}^{++}\right.$and $\left.\mathrm{Mg}^{++}\right)$. Primary antibody incubation was done in dilution buffer $\left(10 \%\right.$ blocking buffer), overnight at $4^{\circ} \mathrm{C}$. Secondary antibody (Sigma Aldrich, MO, USA) incubation was done in dilution buffer at room temperature for an hour. After $2 \mathrm{x}$ washes in DPBS $\left(\mathrm{w} / \mathrm{Ca}^{++}\right.$and $\mathrm{Mg}^{++}$) cells were stained with Hoechst (1:2000) in dilution buffer. Cells were washed $3 x$ and imaged with Imagexpress Micro (Molecular Devices, CA, USA). Quantification of astrocytic markers and astrocytic processes was done by using the Multi-Wavelength Cell Scoring and Neurite Outgrowth module, respectively. A detailed list of the antibodies used can be found in Table S1. 


\section{mRNA expression analysis}

Cells were collected, lysed and total RNA was extracted using the RNeasy Mini kit (Qiagen, Germany); cDNA synthesis was carried out using the High-capacity RNA-to-cDNA kit on a thermal cycler (VWR, PA, USA). cDNA samples were analyzed using MySpec (VWR, PA, USA). TaqMan probes of interest were incubated with cDNA samples in Fast Advanced Master Mix. For all samples, GAPDH TaqMan probes were included as reference. Samples were run on a BioRad CFX96 Touch Real-Time PCR Detection system using the multiplex option for superior Ct quantification. Depending on the graph presented, either $\Delta C t$ or $\Delta \triangle \mathrm{Cts}$ were calculated. Samples that lacked Ct values (e.g., RELN, GFAP for all NES lines) or had values over 35 were assigned a $C t$ value of 35 for the $\Delta \Delta C t$ analysis to be possible (or to minimize overestimation of results). PCA was done with R using the prcomp function (R Core Team (2021). R: A language and environment for statistical computing. R Foundation for Statistical Computing, Vienna, Austria. URL https://www.R-project.org/) and hierarchical clustering with the Morpheus software using average linkage clustering, person correlation (Morpheus, https://software.broadinstitute.org/morpheus). A list of the TaqMan probes used can be found in Table S2.

\section{Glutamate uptake}

NES C9, hiAstrocytes C9 and HFA were seeded out in 96-well plates in their respective media. After $72 \mathrm{~h}$ cells were washed once with HBSS $\left(\mathrm{w} / \mathrm{Ca}^{++}\right.$and $\mathrm{Mg}^{++}$) and then incubated in either vehicle (DMSO) or inhibitors UCPH1 (1UM, EAAT1 inhibitor, Abcam, UK) and WAY 213613 (1.5uM, EAAT2 inhibitor, R\&D Systems, MN, USA) for $30 \mathrm{~min}$ in HBSS ( $\mathrm{w} / \mathrm{Ca}^{++}$and $\mathrm{Mg}^{++}$). After that, HBSS was changed with HBSS ( $w$ / $\mathrm{Ca}^{++}$and $\mathrm{Mg}^{++}$) containing 50uM of glutamic acid with either vehicle (DMSO) or inhibitors and incubated for $60 \mathrm{~min}$. Samples were taken and analyzed with the glutamate assay kit (Abcam, UK). Following sample collection, cells were incubated with Image-IT ${ }^{T M}$ DEAD Green ${ }^{T M}$. Cells were consequently fixed and stained with Hoechst. Images were captured within 24-48h with ImageXpress Micro 10x (Molecular Devices, CA, USA). Live dead count was assessed by the Live/Dead module.

\section{Inflammatory assay}

NES, hiAstrocytes and HFA were seeded out in 96-well plates, and 48h after cells were washed once with DPBS ( $\mathrm{w} / \mathrm{Ca}^{++}$and $\mathrm{Mg}^{++}$) and challenged with IL-1 $(50 \mathrm{ng} / \mathrm{ml}, \mathrm{R} \& D$ Systems, MN, USA). For the basal conditions, cells were washed with DPBS ( $w / \mathrm{Ca}^{++}$and $\mathrm{Mg}^{++}$), and media was replenished. After 24h, samples were collected and snap-frozen. Samples were analysed using the Mesoscale system (U-PLEX plate) according to kit instructions. The condition termed "IL-1 $324 \mathrm{~h}$ " for analysis of ICAM-1 also followed the same procedure. Cell numbers were determined in the same procedure as in the glutamate assay.

\section{Glutathione assay}

NES, hiAstrocytes and HFA were seeded out in T12.5 flasks in their respective media for 48h; cells were washed with ( $\mathrm{w} / \mathrm{Ca++}$ and $\mathrm{Mg}++$ ) all cells were changed to N2B27 with low EGF $(1 \mathrm{ng} / \mathrm{ml}$ ) and B27 without antioxidants, control media was also included in a cell-free T12.5 flask. After $24 \mathrm{~h}$, media samples were collected from all cells and control media, and cells were harvested and snap-frozen. Media samples and cell suspensions were analyzed kinetically with the Glutathione assay kit (Sigma Aldrich, MO, USA) according to the manufacturer's instructions with the following modifications: the kinetic reactions were analyzed for $30 \mathrm{~min}$ and the calibration curve was adjusted to include more 
points. For extracellular quantification, the cell-free media flask values were subtracted from the media samples. Deproteinized samples were reconstituted in $0.5 \mathrm{M}$, and following complete reconstitution, samples were diluted to $100 \mathrm{mM} \mathrm{NaOH}$, and total protein content was measured using the BCA kit.

The cell number/mg protein ratio was calculated on a separate occasion by manually counting cells through a hemocytometer (each sample was counted twice. For each count, four squares were analyzed). A known number of cells were lysed according to the Glutathione assay kit procedure described above. Deproteinized samples were reconstituted and analyzed as described above.

\section{References}

Akiyama, H. et al. (1993) 'Expression of intercellular adhesion molecule (ICAM)-1 by a subset of astrocytes in Alzheimer disease and some other degenerative neurological disorders', Acta Neuropathologica, 85(6), pp. 628-634. doi: 10.1007/BF00334673.

Araque, A. et al. (1999) 'Tripartite synapses: glia, the unacknowledged partner', Trends in Neurosciences, 22(5), pp. 208-215. doi: 10.1016/S0166-2236(98)01349-6.

Bains, J. S. and Shaw, C. A. (1997) 'Neurodegenerative disorders in humans: the role of glutathione in oxidative stress-mediated neuronal death', Brain Research. Brain Research Reviews, 25(3), pp. 335-358. doi: 10.1016/s0165-0173(97)00045-3.

Bar-Peled, O. et al. (1997) 'Distribution of Glutamate Transporter Subtypes During Human Brain Development', Journal of Neurochemistry, 69(6), pp. 2571-2580. doi: https://doi.org/10.1046/j.14714159.1997.69062571.x.

Barres, B. A. (2008) 'The Mystery and Magic of Glia: A Perspective on Their Roles in Health and Disease', Neuron, 60(3), pp. 430-440. doi: 10.1016/j.neuron.2008.10.013.

Bradley, R. A. et al. (2019) 'Regionally specified human pluripotent stem cell-derived astrocytes exhibit different molecular signatures and functional properties', Development, 146(dev170910). doi: 10.1242/dev.170910.

Bristot Silvestrin, R. et al. (2013) 'Animal model of autism induced by prenatal exposure to valproate: Altered glutamate metabolism in the hippocampus', Brain Research, 1495, pp. 52-60. doi: 10.1016/j.brainres.2012.11.048.

Brosnan, C. F. et al. (1995) 'Cytokine localization in multiple sclerosis lesions: Correlation with adhesion molecule expression and reactive nitrogen species', Neurology, 45(6 Suppl 6), pp. S16-S21. doi: 10.1212/WNL.45.6_Suppl_6.S16.

Cadet, J. L. and Brannock, C. (1998) 'Invited Review Free radicals and the pathobiology of brain dopamine systems', Neurochemistry International, 32(2), pp. 117-131. doi: 10.1016/S01970186(97)00031-4.

Chambers, S. M. et al. (2009) 'Highly efficient neural conversion of human ES and iPS cells by dual inhibition of SMAD signaling', Nature biotechnology, 27(3), pp. 275-280. doi: 10.1038/nbt.1529. 
Choi, S. S. et al. (2014) 'Human Astrocytes: Secretome Profiles of Cytokines and Chemokines', PLOS ONE, 9(4), p. e92325. doi: 10.1371/journal.pone.0092325.

Clarke, B. E. et al. (2021) 'Regionally encoded functional heterogeneity of astrocytes in health and disease: A perspective', Glia, 69(1), pp. 20-27. doi: https://doi.org/10.1002/glia.23877.

Codeluppi, S. et al. (2011) 'Influence of rat substrain and growth conditions on the characteristics of primary cultures of adult rat spinal cord astrocytes', Journal of Neuroscience Methods, 197(1), pp. 118127. doi: 10.1016/j.jneumeth.2011.02.011.

Colton, C. K. et al. (2010) 'Identification of translational activators of glial glutamate transporter EAAT2 through cell-based high-throughput screening: an approach to prevent excitotoxicity', Journal of Biomolecular Screening, 15(6), pp. 653-662. doi: 10.1177/1087057110370998.

DeSilva, T. M. et al. (2012) 'Expression of EAAT2 in Neurons and Protoplasmic Astrocytes during Human Cortical Development', The Journal of comparative neurology, 520(17), pp. 3912-3932. doi: 10.1002/cne.23130.

Devesa, A. et al. (1993) 'Glutathione metabolism in primary astrocyte cultures: flow cytometric evidence of heterogeneous distribution of GSH content', Brain Research, 618(2), pp. 181-189. doi: 10.1016/00068993(93)91264-S.

Dringen, R. (2000) 'Metabolism and functions of glutathione in brain', Progress in Neurobiology, 62(6), pp. 649-671. doi: 10.1016/S0301-0082(99)00060-X.

Dringen, R. and Hamprecht, B. (1998) 'Glutathione Restoration as Indicator for Cellular Metabolism of Astroglial Cells', Developmental Neuroscience, 20(4-5), pp. 401-407. doi: 10.1159/000017337.

Dringen, R., Kranich, O. and Hamprecht, B. (1997) 'The $\gamma$-Glutamyl Transpeptidase Inhibitor Acivicin Preserves Glutathione Released by Astroglial Cells in Culture', Neurochemical Research, 22(6), pp. 727733. doi: 10.1023/A:1027310328310.

Falk, A. et al. (2012) 'Capture of Neuroepithelial-Like Stem Cells from Pluripotent Stem Cells Provides a Versatile System for In Vitro Production of Human Neurons', PLOS ONE, 7(1), p. e29597. doi: 10.1371/journal.pone.0029597.

Fang, C. et al. (2017) 'The Interrelation between Reactive Oxygen Species and Autophagy in Neurological Disorders', Oxidative Medicine and Cellular Longevity, 2017. doi: 10.1155/2017/8495160.

Frohman, E. M. et al. (1989) 'The induction of intercellular adhesion molecule 1 (ICAM-1) expression on human fetal astrocytes by interferon- $\lambda$, tumor necrosis factor $\alpha$, lymphotoxin, and interleukin-1: relevance to intracerebral antigen presentation', Journal of Neuroimmunology, 23(2), pp. 117-124. doi: 10.1016/0165-5728(89)90030-1.

Garcia-Esparcia, P. et al. (2018) 'Glutamate Transporter GLT1 Expression in Alzheimer Disease and Dementia With Lewy Bodies', Frontiers in Aging Neuroscience, 10. doi: 10.3389/fnagi.2018.00122. 
Gu, F. et al. (2013) 'Alterations in mitochondrial DNA copy number and the activities of electron transport chain complexes and pyruvate dehydrogenase in the frontal cortex from subjects with autism', Translational Psychiatry, 3(9), p. e299. doi: 10.1038/tp.2013.68.

Harris, J. L. et al. (2012) 'Altered neurochemical profile after traumatic brain injury: 1H-MRS biomarkers of pathological mechanisms', Journal of Cerebral Blood Flow \& Metabolism, 32(12), pp. 2122-2134. doi: 10.1038/jcbfm.2012.114.

Haselbacher, G. et al. (1989) 'Long-term cultivation of cryopreserved human fetal brain cells in a chemically defined medium', Journal of Neuroscience Methods, 30(2), pp. 121-131. doi: 10.1016/01650270(89)90058-7.

Heck, N. et al. (2003) 'Astrocytes in culture express fibrillar collagen', Glia, 41(4), pp. 382-392. doi: 10.1002/glia.10184.

Heck, N. et al. (2007) 'Evidence for distinct leptomeningeal cell-dependent paracrine and EGF-linked autocrine regulatory pathways for suppression of fibrillar collagens in astrocytes', Molecular and Cellular Neuroscience, 36(1), pp. 71-85. doi: 10.1016/j.mcn.2007.06.002.

Héry, C. et al. (1995) 'Adhesion to human neurons and astrocytes of monocytes: the role of interaction of CR3 and ICAM-1 and modulation by cytokines', Journal of Neuroimmunology, 57(1-2), pp. 101-109. doi: 10.1016/0165-5728(94)00168-n.

Hirrlinger, J., Schulz, J. B. and Dringen, R. (2002) 'Glutathione release from cultured brain cells: Multidrug resistance protein 1 mediates the release of GSH from rat astroglial cells', Journal of Neuroscience Research, 69(3), pp. 318-326. doi: https://doi.org/10.1002/jnr.10308.

Hochstim, C. et al. (2008) 'Identification of Positionally Distinct Astrocyte Subtypes whose Identities Are Specified by a Homeodomain Code', Cell, 133(3), pp. 510-522. doi: 10.1016/j.cell.2008.02.046.

Holmqvist, S. et al. (2015) 'Generation of human pluripotent stem cell reporter lines for the isolation of and reporting on astrocytes generated from ventral midbrain and ventral spinal cord neural progenitors', Stem Cell Research, 15(1), pp. 203-220. doi: 10.1016/j.scr.2015.05.014.

Huang, S.-F. et al. (2020) 'Astrocyte glutathione maintains endothelial barrier stability', Redox Biology, 34, p. 101576. doi: 10.1016/j.redox.2020.101576.

Kele, M. et al. (2016) 'Generation of human iPS cell line CTL07-II from human fibroblasts, under defined and xeno-free conditions', Stem Cell Research, 17(3), pp. 474-478. doi: 10.1016/j.scr.2016.09.028.

Lam, M., Moslem, M., et al. (2019) 'Single cell analysis of autism patient with bi-allelic NRXN1-alpha deletion reveals skewed fate choice in neural progenitors and impaired neuronal functionality', Experimental Cell Research, 383(1), p. 111469. doi: 10.1016/j.yexcr.2019.06.014.

Lam, M., Sanosaka, T., et al. (2019) 'Single-cell study of neural stem cells derived from human iPSCs reveals distinct progenitor populations with neurogenic and gliogenic potential', Genes to Cells, 24(12), pp. 836-847. doi: https://doi.org/10.1111/gtc.12731. 
Lange, S. C. et al. (2012) 'Primary cultures of astrocytes: Their value in understanding astrocytes in health and disease', Neurochemical research, 37(11), pp. 2569-2588. doi: 10.1007/s11064-012-0868-0.

Lee, M. L. et al. (2017) 'Brain endothelial cells induce astrocytic expression of the glutamate transporter GLT-1 by a Notch-dependent mechanism', Journal of Neurochemistry, 143(5), pp. 489-506. doi: 10.1111/jnc.14135.

Lee, S. J. et al. (2000) 'ICAM-1-Induced Expression of Proinflammatory Cytokines in Astrocytes: Involvement of Extracellular Signal-Regulated Kinase and p38 Mitogen-Activated Protein Kinase Pathways', The Journal of Immunology, 165(8), pp. 4658-4666. doi: 10.4049/jimmunol.165.8.4658.

Lee, S.-G. et al. (2008) 'Mechanism of Ceftriaxone Induction of Excitatory Amino Acid Transporter-2 Expression and Glutamate Uptake in Primary Human Astrocytes', The Journal of Biological Chemistry, 283(19), pp. 13116-13123. doi: 10.1074/jbc.M707697200.

Leventoux, N. et al. (2020) 'Human Astrocytes Model Derived from Induced Pluripotent Stem Cells', Cells, 9(12), p. 2680. doi: 10.3390/cells9122680.

Li, J. et al. (2013) 'Oxidative Stress and Neurodegenerative Disorders', International Journal of Molecular Sciences, 14(12), pp. 24438-24475. doi: 10.3390/ijms141224438.

$\mathrm{Li}$, J. et al. (2019) 'Astrocyte-to-astrocyte contact and a positive feedback loop of growth factor signaling regulate astrocyte maturation', Glia, 67(8), pp. 1571-1597. doi: https://doi.org/10.1002/glia.23630.

Liddelow, S. A. et al. (2017) 'Neurotoxic reactive astrocytes are induced by activated microglia', Nature, 541(7638), pp. 481-487. doi: 10.1038/nature21029.

Lovell, M. A., Xie, C. and Markesbery, W. R. (1998) 'Decreased glutathione transferase activity in brain and ventricular fluid in Alzheimer's disease', Neurology, 51(6), pp. 1562-1566. doi:

10.1212/WNL.51.6.1562.

Lundin, A. et al. (2018) 'Human iPS-Derived Astroglia from a Stable Neural Precursor State Show Improved Functionality Compared with Conventional Astrocytic Models', Stem Cell Reports, 10(3), pp. 1030-1045. doi: 10.1016/j.stemcr.2018.01.021.

Lundin, A. et al. (2020) 'hiPS-Derived Astroglia Model Shows Temporal Transcriptomic Profile Related to Human Neural Development and Glia Competence Acquisition of a Maturing Astrocytic Identity', Advanced Biosystems, 4(5), p. 1900226. doi: https://doi.org/10.1002/adbi.201900226.

Ma, W. et al. (2008) 'Cell-extracellular matrix interactions regulate neural differentiation of human embryonic stem cells', BMC Developmental Biology, 8(1), p. 90. doi: 10.1186/1471-213X-8-90.

Michler-Stuke, A., Wolff, J. R. and Bottenstein, J. E. (1984) 'Factors influencing astrocyte growth and development in defined media', International Journal of Developmental Neuroscience, 2(6), pp. 575584. doi: 10.1016/0736-5748(84)90035-2.

Minich, T. et al. (2006) 'The multidrug resistance protein 1 (Mrp1), but not Mrp5, mediates export of glutathione and glutathione disulfide from brain astrocytes', Journal of Neurochemistry, 97(2), pp. 373384. doi: https://doi.org/10.1111/j.1471-4159.2006.03737.x. 
Müller, N. (2019) 'The Role of Intercellular Adhesion Molecule-1 in the Pathogenesis of Psychiatric Disorders', Frontiers in Pharmacology, 10. doi: 10.3389/fphar.2019.01251.

Nadadhur, A. G. et al. (2018) 'Patterning factors during neural progenitor induction determine regional identity and differentiation potential in vitro', Stem Cell Research, 32, pp. 25-34. doi:

10.1016/j.scr.2018.08.017.

Oberheim, N. A. et al. (2008) 'Loss of astrocytic domain organization in the epileptic brain', The Journal of Neuroscience: The Official Journal of the Society for Neuroscience, 28(13), pp. 3264-3276. doi: 10.1523/JNEUROSCI.4980-07.2008.

Oberheim, N. A. et al. (2009) 'Uniquely Hominid Features of Adult Human Astrocytes', The Journal of Neuroscience, 29(10), pp. 3276-3287. doi: 10.1523/JNEUROSCI.4707-08.2009.

Oksanen, M. et al. (2017) 'PSEN1 Mutant iPSC-Derived Model Reveals Severe Astrocyte Pathology in Alzheimer's Disease', Stem Cell Reports, 9(6), pp. 1885-1897. doi: 10.1016/j.stemcr.2017.10.016.

Pajarillo, E. et al. (2019) 'The role of astrocytic glutamate transporters GLT-1 and GLAST in neurological disorders: potential targets for neurotherapeutics', Neuropharmacology, 161, p. 107559. doi: 10.1016/j.neuropharm.2019.03.002.

Perea, G., Navarrete, M. and Araque, A. (2009) 'Tripartite synapses: astrocytes process and control synaptic information', Trends in Neurosciences, 32(8), pp. 421-431. doi: 10.1016/j.tins.2009.05.001.

Perriot, S. et al. (2018) 'Human Induced Pluripotent Stem Cell-Derived Astrocytes Are Differentially Activated by Multiple Sclerosis-Associated Cytokines', Stem Cell Reports, 11(5), pp. 1199-1210. doi: 10.1016/j.stemcr.2018.09.015.

Placone, A. F. et al. (2015) 'Human Astrocytes Develop Physiological Morphology and Remain Quiescent in a Novel 3D Matrix', Biomaterials, 42, pp. 134-143. doi: 10.1016/j.biomaterials.2014.11.046.

Popa-Wagner, A. et al. (2013) 'ROS and Brain Diseases: The Good, the Bad, and the Ugly', Oxidative Medicine and Cellular Longevity, 2013, p. e963520. doi: 10.1155/2013/963520.

Raponi, E. et al. (2007) 'S100B expression defines a state in which GFAP-expressing cells lose their neural stem cell potential and acquire a more mature developmental stage', Glia, 55(2), pp. 165-177. doi: 10.1002/glia.20445.

Raps, S. P. et al. (1989) 'Glutathione is present in high concentrations in cultured astrocytes but not in cultured neurons', Brain Research, 493(2), pp. 398-401. doi: 10.1016/0006-8993(89)91178-5.

Renes, J. et al. (1999) 'ATP- and glutathione-dependent transport of chemotherapeutic drugs by the multidrug resistance protein MRP1', British Journal of Pharmacology, 126(3), pp. 681-688. doi: 10.1038/sj.bjp.0702360.

Rodríguez, J. J. et al. (2009) 'Astroglia in dementia and Alzheimer's disease', Cell Death \& Differentiation, 16(3), pp. 378-385. doi: 10.1038/cdd.2008.172. 
Rose, S. et al. (2012) 'Evidence of oxidative damage and inflammation associated with low glutathione redox status in the autism brain', Translational Psychiatry, 2(7), p. e134. doi: 10.1038/tp.2012.61.

Rosenblum, L. T. and Trotti, D. (2017) 'EAAT2 and the molecular signature of amyotrophic lateral sclerosis', Advances in neurobiology, 16, pp. 117-136. doi: 10.1007/978-3-319-55769-4_6.

Rothstein, J. D. et al. (1995) 'Selective loss of glial glutamate transporter GLT-1 in amyotrophic lateral sclerosis', Annals of Neurology, 38(1), pp. 73-84. doi: https://doi.org/10.1002/ana.410380114.

Rothstein, J. D. et al. (2005) 'Beta-lactam antibiotics offer neuroprotection by increasing glutamate transporter expression', Nature, 433(7021), pp. 73-77. doi: 10.1038/nature03180.

Rowitch, D. H. and Kriegstein, A. R. (2010) 'Developmental genetics of vertebrate glial-cell specification', Nature, 468(7321), pp. 214-222. doi: 10.1038/nature09611.

Roybon, L. et al. (2013) ‘Human Stem Cell-Derived Spinal Cord Astrocytes with Defined Mature or Reactive Phenotypes', Cell Reports, 4(5), pp. 1035-1048. doi: 10.1016/j.celrep.2013.06.021.

Sabbagh, M. F. et al. (2018) 'Transcriptional and epigenomic landscapes of CNS and non-CNS vascular endothelial cells', eLife. Edited by E. Dejana, D. Y. Stainier, and E. Dejana, 7, p. e36187. doi:

10.7554/elife.36187.

Sagara, J., Makino, N. and Bannai, S. (1996) 'Glutathione Efflux from Cultured Astrocytes', Journal of Neurochemistry, 66(5), pp. 1876-1881. doi: https://doi.org/10.1046/j.1471-4159.1996.66051876.x.

Santos, R. et al. (2017) 'Differentiation of Inflammation-Responsive Astrocytes from Glial Progenitors Generated from Human Induced Pluripotent Stem Cells', Stem Cell Reports, 8(6), pp. 1757-1769. doi: 10.1016/j.stemcr.2017.05.011.

Savchenko, E. et al. (2019) 'FGF family members differentially regulate maturation and proliferation of stem cell-derived astrocytes', Scientific Reports, 9. doi: 10.1038/s41598-019-46110-1.

Schiweck, J., Eickholt, B. J. and Murk, K. (2018) 'Important Shapeshifter: Mechanisms Allowing Astrocytes to Respond to the Changing Nervous System During Development, Injury and Disease', Frontiers in Cellular Neuroscience, 12. doi: 10.3389/fncel.2018.00261.

Schlag, B. D. et al. (1998) 'Regulation of the glial Na+-dependent glutamate transporters by cyclic AMP analogs and neurons', Molecular Pharmacology, 53(3), pp. 355-369. doi: 10.1124/mol.53.3.355.

Shaltouki, A. et al. (2013) 'Efficient Generation of Astrocytes from Human Pluripotent Stem Cells in Defined Conditions', STEM CELLS, 31(5), pp. 941-952. doi: https://doi.org/10.1002/stem.1334.

Sinyuk, M. and Williams, J. L. (2020) 'Dissection and Isolation of Murine Glia from Multiple Central Nervous System Regions', JoVE (Journal of Visualized Experiments), (160), p. e61345. doi: $10.3791 / 61345$.

Soubannier, V. et al. (2020) 'Characterization of human iPSC-derived astrocytes with potential for disease modeling and drug discovery', Neuroscience Letters, 731, p. 135028. doi:

10.1016/j.neulet.2020.135028. 
Sriram, S. and Steiner, I. (2005) 'Experimental allergic encephalomyelitis: a misleading model of multiple sclerosis', Annals of Neurology, 58(6), pp. 939-945. doi: 10.1002/ana.20743.

Sultana, R. and Butterfield, D. A. (2004) 'Oxidatively Modified GST and MRP1 in Alzheimer's Disease Brain: Implications for Accumulation of Reactive Lipid Peroxidation Products', Neurochemical Research, 29(12), pp. 2215-2220. doi: 10.1007/s11064-004-7028-0.

Swanson, R. A. et al. (1997) 'Neuronal regulation of glutamate transporter subtype expression in astrocytes', The Journal of Neuroscience: The Official Journal of the Society for Neuroscience, 17(3), pp. 932-940.

't Hart, B. A. (2016) 'Why does multiple sclerosis only affect human primates?', Multiple Sclerosis Journal, 22(4), pp. 559-563. doi: 10.1177/1352458515591862.

Takahashi, K. et al. (2015) 'Restored glial glutamate transporter EAAT2 function as a potential therapeutic approach for Alzheimer's disease', The Journal of Experimental Medicine, 212(3), pp. 319332. doi: $10.1084 /$ jem.20140413.

Tchieu, J. et al. (2019) 'NFIA is a gliogenic switch enabling rapid derivation of functional human astrocytes from pluripotent stem cells', Nature Biotechnology, 37(3), pp. 267-275. doi: 10.1038/s41587019-0035-0.

Tcw, J. et al. (2017) 'An Efficient Platform for Astrocyte Differentiation from Human Induced Pluripotent Stem Cells', Stem Cell Reports, 9(2), pp. 600-614. doi: 10.1016/j.stemcr.2017.06.018.

Uhlin, E. et al. (2017) 'Derivation of human iPS cell lines from monozygotic twins in defined and xeno free conditions', Stem Cell Research, 18, pp. 22-25. doi: 10.1016/j.scr.2016.12.006.

Wang, X. F. and Cynader, M. S. (2000) 'Astrocytes Provide Cysteine to Neurons by Releasing Glutathione', Journal of Neurochemistry, 74(4), pp. 1434-1442. doi: https://doi.org/10.1046/j.14714159.2000.0741434.x.

Windrem, M. S. et al. (2017) 'Human iPSC Glial Mouse Chimeras Reveal Glial Contributions to Schizophrenia', Cell Stem Cell, 21(2), pp. 195-208.e6. doi: 10.1016/j.stem.2017.06.012.

Yudkoff, M. et al. (1990) 'Glutathione Turnover in Cultured Astrocytes: Studies with [15N]Glutamate', Journal of Neurochemistry, 55(1), pp. 137-145. doi: https://doi.org/10.1111/j.1471-

4159.1990.tb08831.x.

Zhang, Y. et al. (2016) 'Purification and Characterization of Progenitor and Mature Human Astrocytes Reveals Transcriptional and Functional Differences with Mouse', Neuron, 89(1), pp. 37-53. doi: 10.1016/j.neuron.2015.11.013.

Zimmermann, D. R. and Dours-Zimmermann, M. T. (2008) 'Extracellular matrix of the central nervous system: from neglect to challenge', Histochemistry and Cell Biology, 130(4), pp. 635-653. doi: 10.1007/s00418-008-0485-9. 
bioRxiv preprint doi: https://doi.org/10.1101/2021.06.07.447336; this version posted June 7, 2021. The copyright holder for this preprint (which was not certified by peer review) is the author/funder. All rights reserved. No reuse allowed without permission. 
Figure 3 hiAstrocytes C9

hiAstrocytes C7

hiAstrocytes AF22

HFA

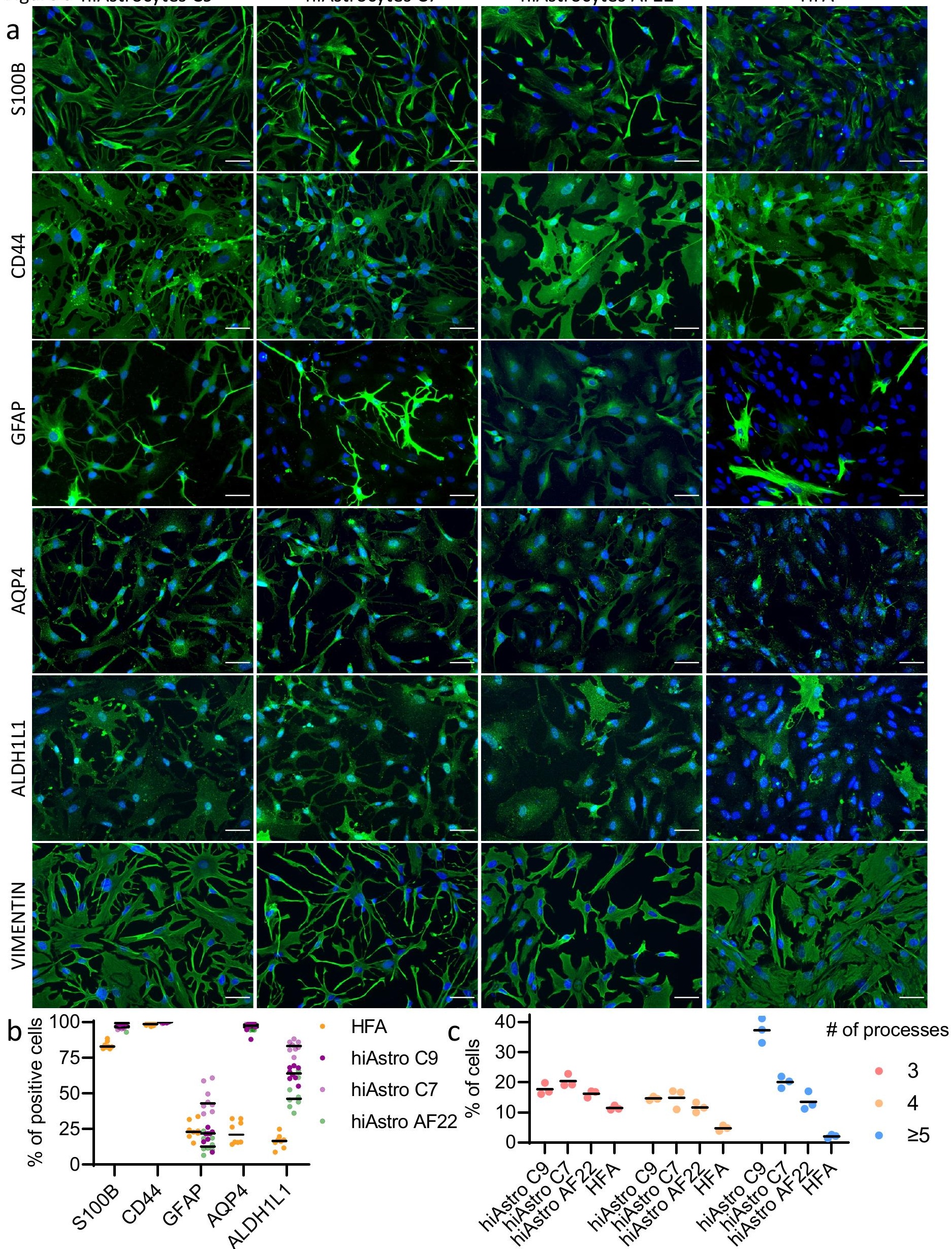

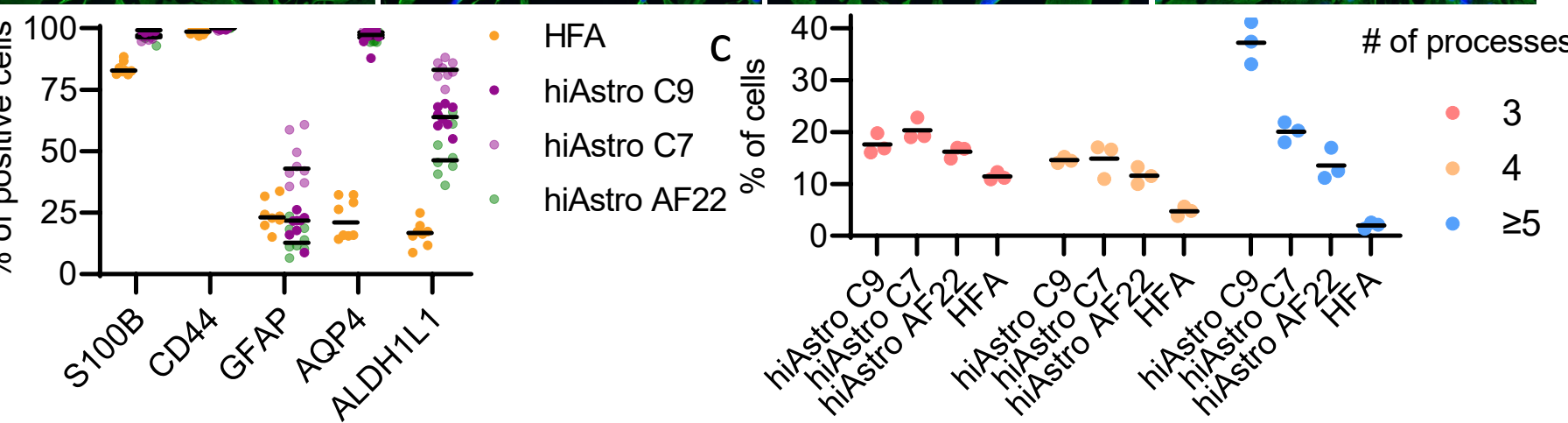



옹
고

๖
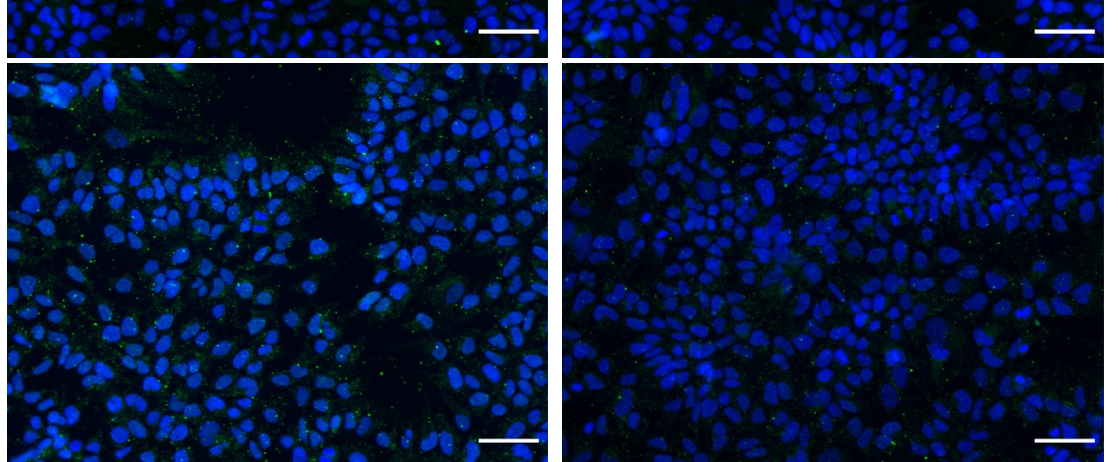

畄

ఫั

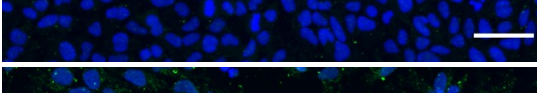

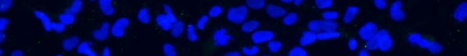
TI

검
몸
룬
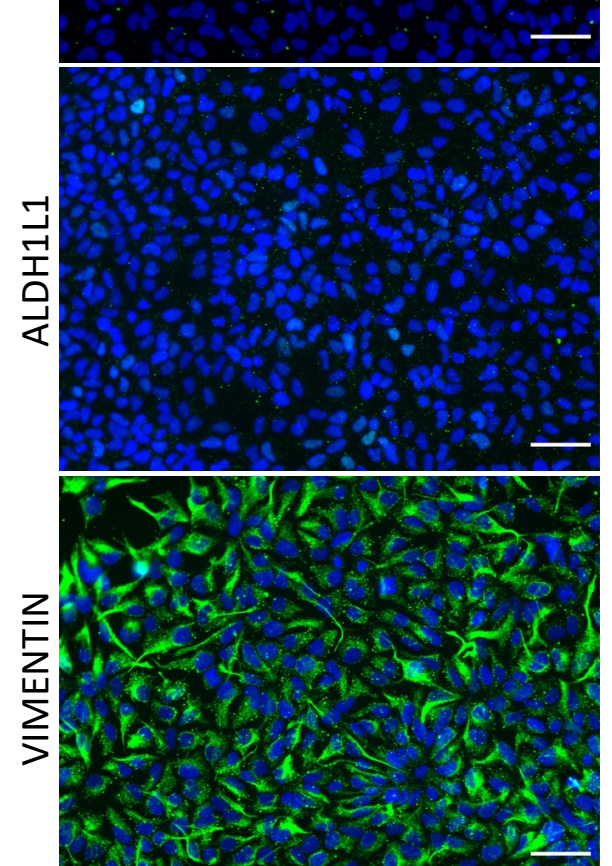

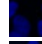

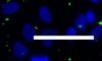
8250 $14 x$

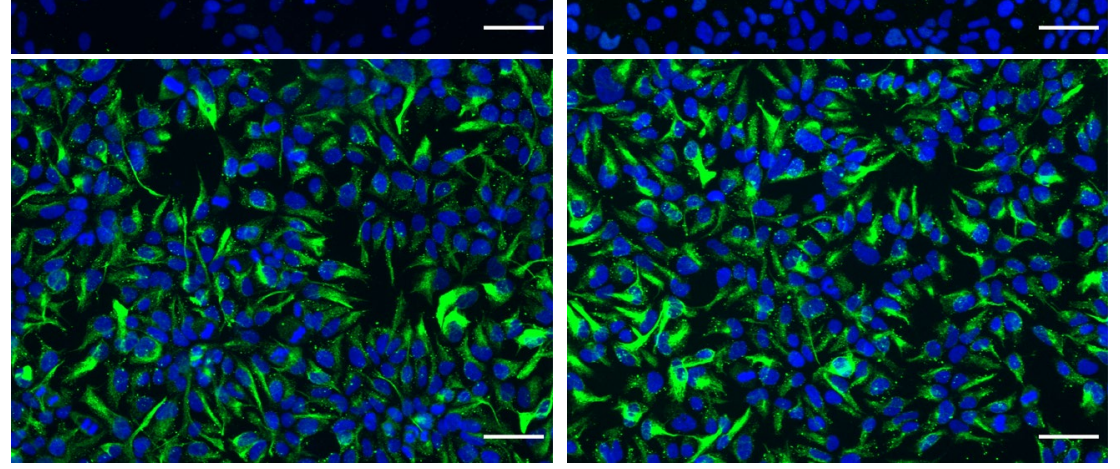


S.Figure 3

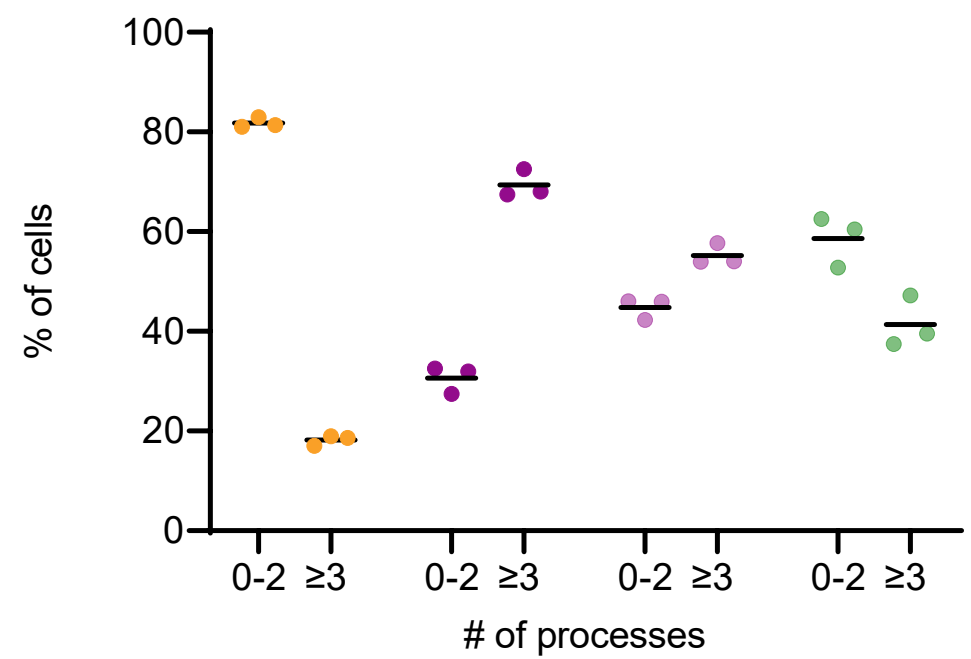

- HFA

- hiAstro C9

- hiAstro C7

- hiAstro AF22 


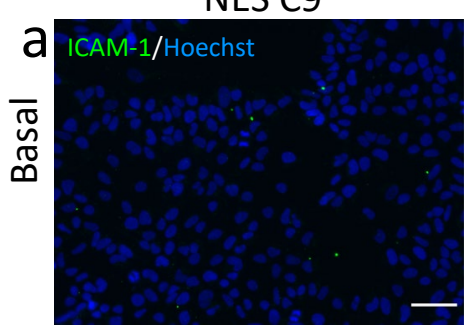

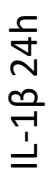

NES C 9

NES C7
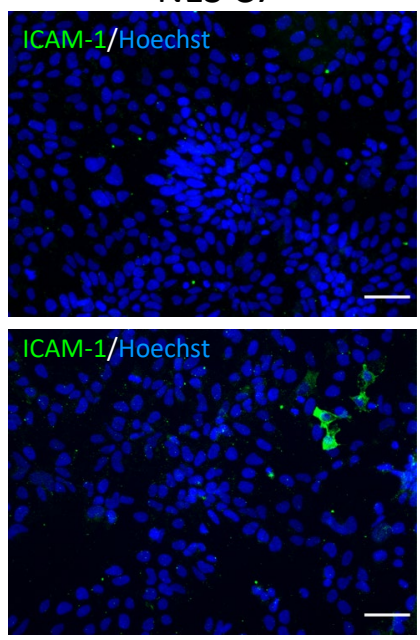

b

$\mathrm{d}$

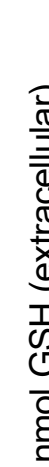

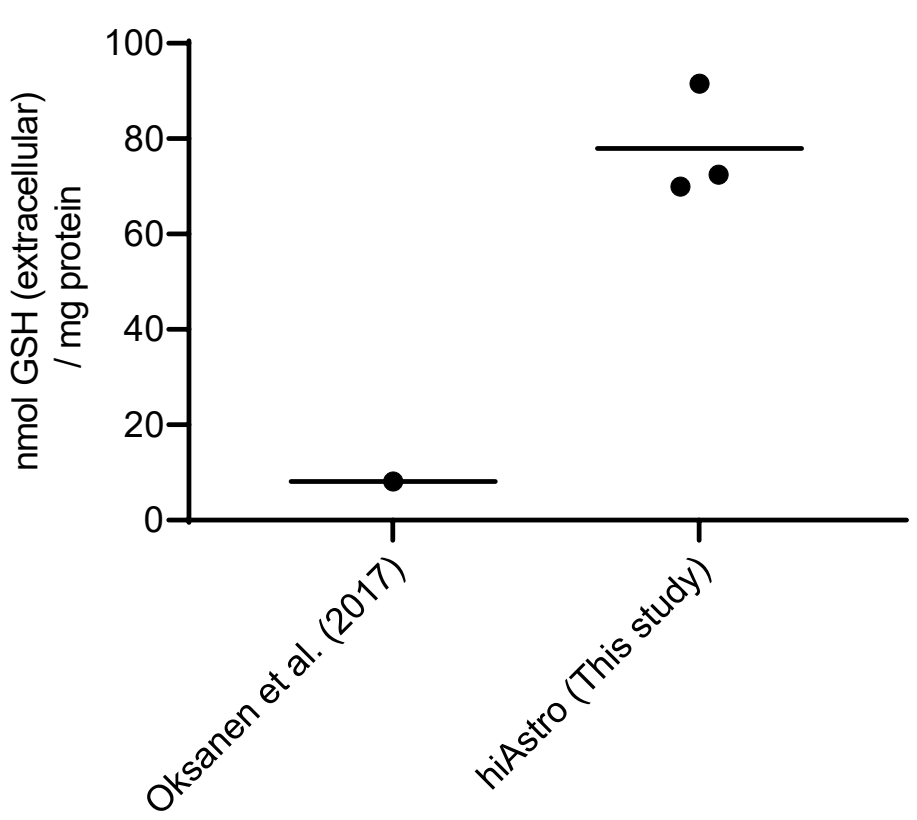

NES AF22
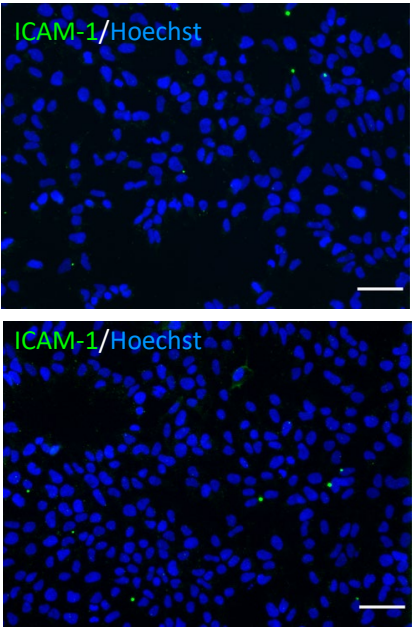

C
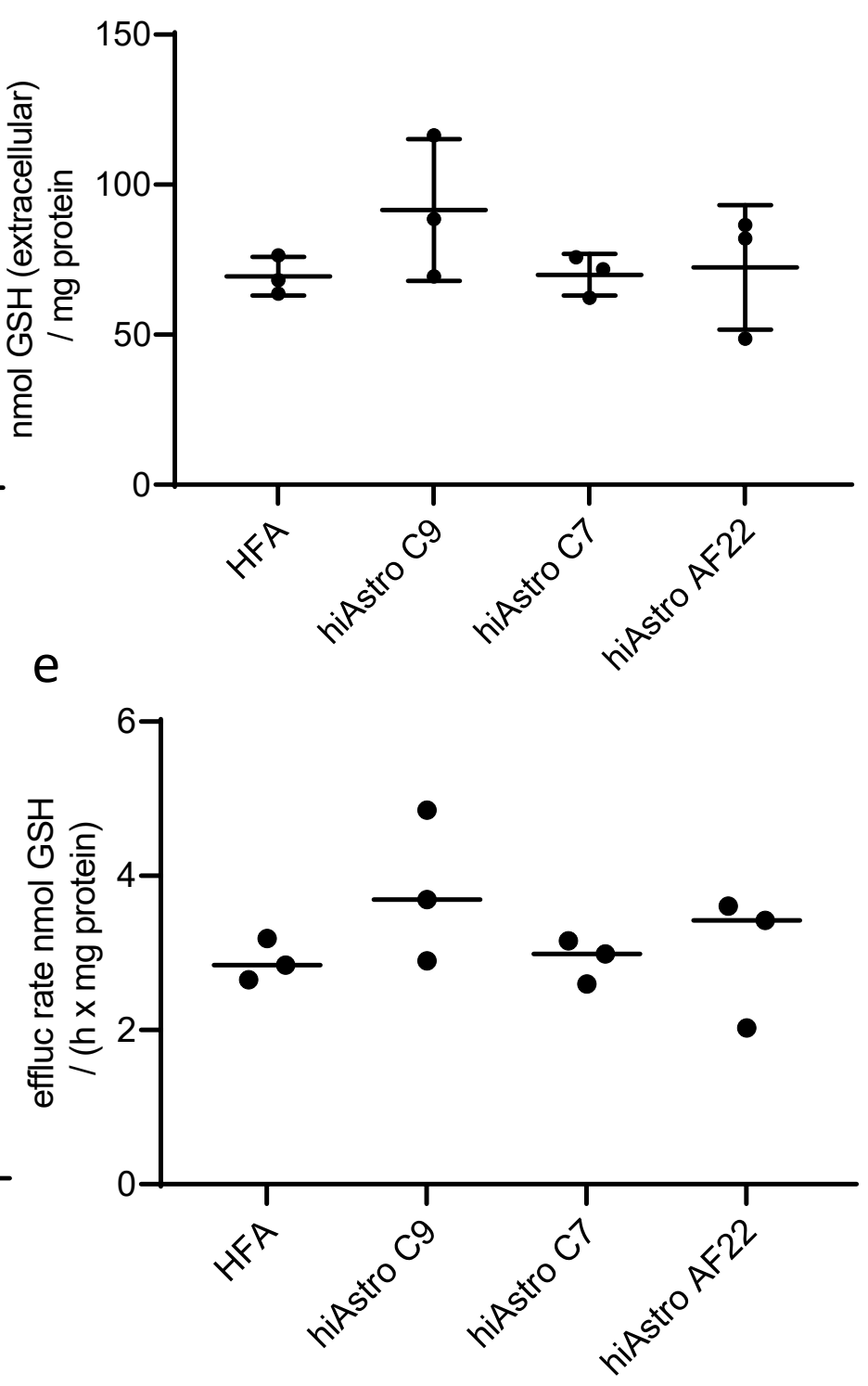


\begin{tabular}{|c|c|c|c|c|}
\hline Antibody & Supplier & Catalog & RRID & Dilution \\
\hline S100B & Abcam & ab52642 & AB_882426 & $1: 100$ \\
\hline CD44 & Abcam & ab157107 & AB_2847859 & $1: 500$ \\
\hline GFAP & DAKO & Z0334 & AB_10013382 & $1: 500$ \\
\hline AQP4 & Sigma-Aldrich & HPA014784 & AB_1844967 & $1: 125$ \\
\hline ALDH1L1 & Abcam & ab190298 & AB_2857848 & $1: 500$ \\
\hline VIMENTIN & Abcam & ab92547 & AB_10562134 & $1: 500$ \\
\hline EAAT1 & $\begin{array}{c}\text { Thermo Fisher } \\
\text { Scientific }\end{array}$ & PA5-19709 & AB_10982702 & $1: 200$ \\
\hline EAAT2 & $\begin{array}{c}\text { Thermo Fisher } \\
\text { Scientific }\end{array}$ & 711020 & AB_2633106 & $1: 250$ \\
\hline ICAM-1 & R\&D Systems & BBA3 & AB_356950 & $1: 50$ \\
\hline
\end{tabular}

Table 2 List of TaqMan probes used in this study.

\begin{tabular}{|l|l|}
\hline \multicolumn{1}{|c|}{ Gene } & \multicolumn{1}{c|}{ TaqMan Assay ID } \\
\hline ALDH1L1 & Hs01003842_m1 \\
\hline CD44 & Hs01075864_m1 \\
\hline DCX & Hs00167057_m1 \\
\hline GAPDH & Hs02758991_g1 \\
\hline GFAP & Hs00909233_m1 \\
\hline NES & Hs04187831_g1 \\
\hline NFIA & Hs00325656_m1 \\
\hline S100B & Hs00902901_m1 \\
\hline SLC1A2 & Hs01102423_m1 \\
\hline SLC1A3 & Hs00904823_g1 \\
\hline SOX1 & Hs01057642_s1 \\
\hline SOX2 & Hs01053049_s1 \\
\hline RELN & Hs01022646_m1 \\
\hline SOX9 & Hs00165814_m1 \\
\hline MAOB & Hs01106246_m1 \\
\hline SLIT1 & Hs00171488_m1 \\
\hline VIM & Hs05024057_m1 \\
\hline GLUL & Hs01013055_g1 \\
\hline AQP4 & Hs00242342_m1 \\
\hline
\end{tabular}

